\title{
Trading Places: What the Research Participant Can Tell the Investigator about Informed Consent
}

\author{
Ann Freeman Cook* and Helena Hoas
}

National Rural Bioethics Project, Department of Psychology - Corbin 343, The University of Montana, Missoula, USA, MT 59812-7397

\begin{abstract}
The issues discussed in this article emerged from a study that explored the decision-making processes used by research participants when deciding to participate in human subjects' research. We discuss the current research and regulatory environment and its influences on participant decision-making. The results of this study create a framework for understanding the challenges of the current informed consent process and offers insights into what may be needed to create an environment that allows research participants to make more enlightened decisions.
\end{abstract}

Keywords: Research ethics; Human subject research; Informed consent; Clinical trial; Institutional review boards; Therapeutic misconception.

Abbreviations: IRB: Institutional Review Board; HIPAA: The Health Insurance Portability and Accountability Act; COI: Conflict of Interest

\section{Introduction}

Article focus - The issues discussed in this article emerged from a 2-year qualitative study that was approved by The University of Montana Institutional Review Board (IRB) and funded by the National Science Foundation. In phase 1 of this 2-year study, the investigators conducted interviews with IRB members from across the country in order to examine the norms and processes that IRBs use when assessing and monitoring the adequacy of the informed consent process $[1,2]$. In phase 2 , the investigators conducted interviews with research subjects in order to explore their experiences with the informed consent process and their subsequent decisions about participating in human subject research. This article, focused on the findings of phase 2, explored the following three research questions: (1) How do participants make decisions when invited to participate, including their experiences of the consent process; (2) what factors influence a decision to enroll in a study; and (3) what information do participants want when invited to enroll in studies? This study contributes to a growing understanding of the decisionmaking processes used by persons who are invited to participate in research and factors that need attention when developing regulations that will optimize protection of human subjects.

\section{Background}

The US federal regulatory system developed to enhance the ability of prospective participants to provide their informed and voluntary consent before participating in research evolved from the work of the National Commission for the Protection of Human Subjects [3]. This commission was convened in 1973 in response to the growing challenges posed by the ethics of experimentation; regulatory guidance for protecting human subjects became codified in the Common Rule in 1991[4]. The Health Insurance Portability and Accountability Act (HIPAA) of 1996 added another layer of protection-related responsibilities [5].

The US federal guidelines stipulate that the consent process for human subjects research has to provide full disclosure of risks and benefits, be understandable, take into consideration participant capacity and competence, and ensure that consent is voluntary [6,7]. Persons must be given information in lay language so that they understand and appreciate the research process and associated risks; consent must be voluntary and those giving their consent must be legally competent to do so [8]. Those standards, although seemingly reasonable, have in practice proven difficult to operationalize. By 1999, Sugarman et al. had identified more than 377 articles and 3,173 hypotheses when conducting a literature review that focused on empirical research studying informed consent and how best to achieve it [9].

In recent years scholars have focused on topics such as participant comprehension and understanding of the research [10-12], how to achieve voluntariness $[13,14]$, and policy issues $[15,16]$. Studies have shown that participants seem to be particularly vulnerable when trying to understand the key research concepts of clinical trials. Often they labor under a therapeutic misconception that leads them to believe that participating in a medical study would provide access to better treatments or diagnoses [17,18]. Huntington et al. [19] claim that although a great deal of thought goes into writing a consent document, a very small number of potential research subjects read, or understand it, in any detailed way. Moreover, few subjects seem to use the informed consent document as a basis for decisions about participation. Huntington also found that both the readability and the length of the consent form can impede true consent [19]. Other scholars assert that research subjects often have an inaccurate or incomplete understanding of research and tend to overestimate the benefits of participation. In part this occurs because terms such as risk, harm, and randomization - terms commonly used in all consent documents may not be well understood by those unfamiliar with research [20]. Boiler plate language required to fulfill an institution's legal obligations may seem confusing and irrelevant to the prospective participant.

To overcome many of the deficits that participants bring to the

*Corresponding author: Ann Freeman Cook, Ph.D. Research Professor, Director National Rural Bioethics Project Department of Psychology - Corbin 341The University of Montana Missoula, USA, MT 59812-7397, Tel: 406243 2467; Fax: 406243 5748; Email: ann.cook@mso.umt.edu

Received October 20, 2011; Accepted December 06, 2011; Published December 10,2011

Citation: Cook AF, Hoas H (2011) Trading Places: What the Research Participant Can Tell the Investigator about Informed Consent. J Clinic Res Bioeth 2:121. doi:10.4172/2155-9627.1000121

Copyright: () 2011 Cook AF, et al. This is an open-access article distributed under the terms of the Creative Commons Attribution License, which permits unrestricted use, distribution, and reproduction in any medium, provided the original author and source are credited. 
consent process, investigators have focused on approaches for making the informed consent process more accessible and understandable. Researchers have been encouraged to use consent forms that reflect 5 th or 8 th or 10 th grade reading levels. Appelbaum and Grisso developed a semi-structured interview instrument to assess the research participants' competence in making informed decisions about participating in clinical research; their instrument is designed to assess skills such as understanding the research and appreciating the consequences of participating [21]. While the literature base continues to expand, the identification of approaches that optimize full disclosure and understanding of the research process remains elusive. Indeed some scholars have suggested that the unfortunate consequence of the top-down regulatory approach and the focus on participant inadequacy seem to have resulted in a system that focuses on the obligation to document compliance, rather than the obligation to truly achieve informed consent from prospective subjects [22,23].

New challenges to the informed consent process may be emerging as clinical research migrates into new environments that engage cadres of new researchers as well as an expanded population of potential research participants. In years past, clinical research was primarily conducted in medical centers or academic medical settings in urban communities. While some clinical research still occurs in such settings, it is increasingly conducted in community hospitals, clinics, and private practice settings, including rural offices where such research engages rural clinicians and their patients. This migration has increased to the point where the vast majority of industry-funded clinical research now occurs in non-academic settings [24-27].

The challenges of conveying complex information in an evolving research environment underscore the need to better understand how participants make decisions when invited to join studies, what factors influence such decisions, and what information the participants want and need when trying to make informed decisions. Such insights may bring us closer to understanding what might be needed to better align the federal guidance with research participants' needs and expectations.

\section{Research Methods}

Sample - Given the exploratory nature of the study a qualitative research approach was used [28-30]. In order to develop the sample of subjects who had participated in biomedical or non-biomedical human subjects' research, a brief description of the study and invitations to participate were sent to selected hospitals, centers, institutes, and research settings across the US. These research sites were selected to be representative of facilities that serve rural populations. Staff at the selected sites then disseminated the information about the study to potential participants who were asked to contact the investigators by phone or email if willing to participate in interviews. This strategy was augmented by a snowball approach [31] whereby research participants contacted others who had been invited to participate in research and had insights to share. Those willing to participate were then scheduled for interviews. After conducting 50 interviews no new information was emerging and so saturation was achieved and the interview process stopped. This decision is consistent with the standards of qualitative research methodology [32,33].

The 50 subjects included those who had participated in clinical trials and other types of research (90\%) as well as those who had either declined or withdrawn (10\%) from human subject research. This resulted in a sample of 33 females (66\%) and 17 males (34\%) from states across the US. The sample was comprised of 39 participants (78\%) over the age of 50 and 11 participants (22\%) below 50 . The higher age of the participants was probably due to the fact that people who are ill or who have health problems are the ones invited to join or seek out participation in clinical trials. These categories of people generally fall in older age cohorts.

The education levels among the participants varied, but were generally high, with $52 \%$ reporting graduate or some graduate training, 35\% some college experience and the remaining $13 \%$ a high school education. The kinds of studies described by the participants included clinical studies such drugs or devices (72\%), psychological, sociological, longitudinal, or behavioral $(46 \%)$, and genetic studies (8\%). These numbers exceed $100 \%$ because some of the participants had participated in more than one study and some studies fit more than one criterion such as a drug study that includes a genetic component.

Data Collection - The interviews were conducted exclusively by the authors, took place either in person or via telephone, and lasted from 45 minutes to one hour. The participant interviews were conducted between July 2009 and July 2010. The design of the semi structured instrument drew on findings from a pilot study that the investigators conducted in 2007 with rural research participants as well as the earlier phase of the investigators' research that focused on the decision-making processes used by IRBs when overseeing research that involves human subjects $[34,35]$. Using semi-structured interview protocols allows the exploration of unanticipated issues and the pursuit of rich examples while adhering to a core set of domains. The core domains used in the second phase of the project are listed in Table 1. Consistent with established qualitative research practices, the interviews continued until data saturation had been achieved, i.e., no new data surfaced. The authors transcribed the interviews verbatim in preparation for qualitative analysis for which Atlas.ti, a qualitative coding software application, was used. The thematic coding was conducted by both authors; it evolved as new themes emerged.

\section{Results}

A sizeable number of representative quotes are provided to showcase the findings of how participants experienced the informed consent process and made decisions about enrollment. The quotes attest to a remarkable homogeneity, among participants, with respect to core beliefs and expectations that influenced or bolstered their decisions.

The consent process - When recounting their experiences with the consent process the participants generally seemed to view the process as a standard or routine activity and did not seem to question either the information or the process itself. Most of the participants (84\%) reported that they had read the informed consent document and that

Experiences with research including invitations to participate

Experience with informed consent forms and procedures

Relationship with the researchers suggesting enrollment

Factors that influence decision to join: illness, compensation, altruism, personal benefits

Role of funding source, purpose of study, and researcher compensation on decisionmaking

Perception of voluntarism (ability to decline or withdraw, 'gentle coercion'?)

Perception of anonymity and confidentiality

Impact of participant vulnerabilities

Impact of perceived risks and benefits

Future willingness to join a research study

Information subjects want and need to make informed decisions

Table 1: Core Domains for Key Informant Interviews. 
they had been given enough time to think about participation before making a decision. Many reported that they had had to initial every page of the consent form and that the person overseeing the consent process underscored the importance of reading and understanding the nature of the research study. Some were given the form in advance and most reported that they had been encouraged to ask questions. Only 3 participants reported that they had felt very rushed during the consent process and felt that they did not get enough time to process the information.

But in spite of these efforts to fully inform, the data show that achieving full understanding of the information provided through the consent process can present a number of serious challenges for research participants. These challenges seem apparent even for participants who, as in this study, are very well educated. As participants appeared to make the decision to join a study before the full consent process was finalized, reading and signing the consent form became a social formality. Consequently, little attention was given to statements about risks and benefits, who was funding the research, or protection of anonymity and confidentiality. For example, $78 \%$ could not remember who funded the research study. Most participants thought that the research provided valuable benefits such as access to state-of-the art medical tests, or the option of getting the best drug. As one respondent explained: "Well more for less. Better care and a better cure. My treatment would be more complete and there would be less chance of reoccurrence." (p47)

Factors influencing decisions to participate - The data show that the participants' decisions were influenced by a number of factors including illness status (66\%), altruism (42\%), and financial benefit (22\%). The potential for personal benefit seemed to play an important role when deciding to enroll in a study. This seemed true particularly when there was a definite benefit like obtaining a drug or device, or some level of monetary compensation. Two thirds of the participants acknowledged that they had received some compensation for participating. When asked about such compensation, twenty-eight percent cited the provision of additional medical tests or medications. This is exemplified by the respondent who said: "But I got the drug - I am sure the drugs are expensive. The one pill was $\$ 150$ each and I took that twice a day." (p.40) Twenty-eight percent cited monetary compensation as a factor in their decision-making. Explained one participant: "When I found out I would get paid, I made up my mind I was going to do it." (p.2) Most of the monetary compensation was quite modest and involved benefits such as reimbursements for parking, travel, or time and effort. Noted one participant: "Sure. You got \$50 up front and $\$ 25$ when they drew blood. That was it. That was their get rich scheme." (p17)

The decisions to participate were also influenced by the mode of recruitment. Forty-six percent of the participants reported that their physician or healthcare provider had suggested they participate, $36 \%$ that they had responded to a newspaper advertisement or a poster, while $28 \%$ said the recommendations had come from friends and family. Three participants (6\%) responded that their participation was mandatory in order to get credit for required course work. Since some participants offered more than one mode of recruitment for their participation in the studies, the numbers do not add up to $100 \%$.

A trusting relationship with a healthcare provider or researcher seems to influence the decisions a prospective human subject makes. Participants generally believed that such a person would have the subject's best interest at heart. Said one participant whose physician had suggested participation: "[It was my] doctor who recruited me. I trust him completely and I love the man." (p.14) others said it was hard to say no if a trusted clinician or a colleague would ask for participation. The need to maintain, not disappoint, or not jeopardize a relationship was strong among the participants. If a participant had responded to an ad about participating, it seemed as if the decision to participate was formed more or less at the time of finding out about the study. Combining intention with opportunity, one participant explained: "I had seen other ads for other research things. I was just curious and we were planning to get a flu shot that week. We were going on the cruise and wanted the protection." (p18)

The ability to withdraw from a study, an important component of achieving true voluntariness, seemed at times to pose difficulties. While most said it would have been easy to withdraw (76\%), many also said that in practice it would feel like "letting the researchers down" or "like ruining the study" (24\%). When asked if it would have been easy to withdraw from a study, a representative quote was as follows: "Oh yeah, at any time. But, I would have felt guilty because I know, as a coordinator you put in a lot of work into the study and into recruiting people and they pull - and then you can't use any of the information." (p12). Explained another: "It would have been easy in terms of the researcher. I would personally feel like a failure and that it would come back to me as karma."(p35)

Appreciation of risks and benefits - Many participants seemed to minimize potential risks and overestimate the benefits of participation. Indeed, eighty-eight percent believed that the benefits of participation outweighed the risks. They believed that participation would bring tangible benefits to oneself or to others and that the risks would be minimal or non-existent. This approach to risk assessment, even in cases where potentially severe risks were clearly stated in the consent documents, is exemplified by the following representative statement: "Because I don't presume there are any risks. They didn't really talk about them. I think that maybe because the form of the medication is a nasal spray it is less intense than pills or injections. Maybe there are fewer risks? I might have been more leery about medication in pill form. Maybe that is naiveté on my part. I guess there is no reason to suspect a nasal spray is less potent." (p38) This statement was made by a welleducated person whose consent document clearly stated that the risks were potentially life threatening. Some participants ignored or refused to even read about potential risks or side effects. This approach is seen in the following comments: "But I didn't read about the side effects in the informed consent form because I don't want to slant my thinking." (p14) Another participant explained: "I thought it was a formality - like what you sign before surgery, the form saying you might die. It was just like the legal language thing and I didn't take it seriously." (p4).

Some participants expressed almost blind trust in the system and believed that if a study is allowed to be conducted and is approved, it must mean that participating is safe and that the risks are minimal. In this context the signing of the consent document was seen more as a formality. Participants offered explanations such as: "I didn't see any risks. I do blood draws regularly and that wasn't scary for me." (p32). Said another: "I knew it [the drug] had come a long way to get to the point where they are doing trials. So I felt comfortable knowing there were no real risks, that it was safe.(p12) Another participant suggested: "I would not be part of a study where someone from out of town comes in to the [local hotel] and recruits local subjects and is around for three weeks. It adds legitimacy that it is run locally." (p18) Explained another: "I admit to being fairly trusting - a philosophy in my life. Perhaps not always good but I do what is in front of me. It was presented in a professional manner in an office setting by a person 
who was articulate." (p19) Said another "Well the only thing I thought was - well they do stents all the time. I can't imagine a stent that would go wrong and maybe the old ones don't work well."(p10). Even a risk statement that identified the potential risk for a heart attack would not deter a participant who trusted the doctor who suggested participation: "Yes. That heart failure was the main risk, but we just felt that it wasn't - well like the doctor said, it is treatable and so we continued. And not knowing the history of the 6 women who got heart failure or what happened to them. So it is hard for us to know what is the best thing to do. And I haven't had a heart problem yet." (p47)

In addition to trust in the system, some also believed that participation would provide access to what would become the new gold standard or the miracle drug. Such was the belief of the person who had severe diabetes and who had responded extremely well to the study drug; he was certain that study participants would be the first to receive prescriptions for the new drug. "I am waiting for the drug to be released. I will be the first patient in [my state] to sign up."(p45) Another participant whose doctor had suggested participation added: "Well, then I started talking myself into it and he [the doctor] said the D-drug would be the new Lipitor, the miracle drug. That sounds so hopeful." (p14) When holding such beliefs, research participants appeared to overlook the consequences of the research design such as randomization, the phase of the clinical trial, and other important factors. This was exemplified by the respondent who explained: "So I thought well if it works, I would be right in line and first to get the full treatment." (p48) Participants also perceived that the additional time and more interaction with healthcare would translate into better treatment and personal benefit such as a cure for their disease. "Well at that time I did, though, at that time I didn't see either benefits or risks. This is how naive I was. I thought maybe if it wasn't a placebo maybe it would cure my osteoporosis." (p16) Participation in a clinical study does indeed provide the opportunity to get more attention from a healthcare provider and often entails more tests than what is usually required. In the case of cancer trials, participation was often perceived as a last resort and the only way to gain access to treatment.

Given the overall approach to risk appraisal, few participants had any understanding, or even awareness, of the risks and challenges of data sharing or management. Persons who participated in genetic studies or longitudinal studies that required release of personal data, including DNA, also minimized risks associated with confidentiality and privacy reporting that they were not particularly concerned about any associated risk. When asked about the study's provision of privacy protection said one participant: "I knew there couldn't be much harm." Another participant in a gene study noticed: "None really. I always worry about privacy issues but they said it was all identified by numbers and privacy would be safe." Regardless of the type of study, the vast majority of participants (81\%) presumed that the protection of privacy was adequate assuming that activities in a "legitimate" setting such as a hospital setting or researcher's office setting would ensure such protection. Neither the understanding of what privacy protection should include nor who would have access to any data were well developed. Said one participant: "It was private. I was the only one. I was the only one in the room." (p7) Others explained when asked about protection of privacy: "Didn't even consider that."(p1) "Yes. They seem to be careful of how they are handling phone calls and the personal upfront interview." (p17)

What participants need and want to know - The semi-structured instrument included questions about what the participants perceived as important information for decision-making regarding participation in research. Included in the instrument were questions such as: what are the most important pieces of information, what do you want to know; what do most people want to know, and were there any surprises? Generally the respondents wanted understandable and relevant information regarding what was required of them and what the "pros and cons" of participating would entail. Said one participant: "I think the risks and the time involvement. And the benefits. What do I get? What is the benefit to me personally? What am I going to get out of this? The results may not help me but maybe I get physicals and transportation and honorarium." (p.5)The vast majority also wanted to make sure that they would not be harmed if they participated in the research. As one participant explained: "I think they want to make sure they are going to be safe and it won't hurt them. They want to know there is some benefit for them. Some do it for totally altruistic reasons but most people want to see some benefit to them. Like trying a new medicine they wouldn't be able to get otherwise. Like, I never had anything to treat this condition before and maybe I will get better if I am on the study. Most people hope they will get better; they hope they won't get the placebo." (p12) Those who participated in clinical trials wanted to make sure that they would get, at minimum, the standard of care. Participation then seemed to provide the added benefit of possible improvements on the current standard of care.

The findings suggest that regardless of background, education level, or age, participants consistently blurred the distinction between research and treatment. While affirming the importance of learning about risks, the participants also believed that they would not be invited to participate if the risks were other than minimal. Most believed that research would provide individual benefit and even optimal personal benefit. Two quotes reflect this orientation; "But I think there is an assumption that you make in a way - that if someone asks you to do this, like if a medical persons asks you - you think they already know the risks are minimal and they would not put you at a great deal of risk. They have your wellbeing at heart. That is how most people would think. But it may not be true. They are doctors. There is the trust thing." (p21) "I think one wants to understand what the study is about. To make sure that information is straightforward. There was nothing about it being double blind, or placebo, or any negative consequences with being part of either group. I would not have participated if it had been random assignment to a group with shot and/or placebo." (p18)

Most participants desired more information about two issues that generally are not fully explained or disclosed during the consent process: (1) the commercial purposes of the research and (2) the extent of researcher or institutional compensation. Regulatory guidelines do not currently stipulate how much information about either issue needs to be disclosed during the consent process. Prior to taking part in the interviews, most participants had not realized that some studies might be designed for commercial purposes, such as extending a patent or developing a "me-too" drug, a drug that replicates an already existing drug. Most of the participants were also unaware of the potential for researcher compensation. Participants thought it was dishonest not to be transparent about researcher compensation and the full purpose of a study and said that hiding such information would not be acceptable. Most (90\%) wanted to know whether a study had such a commercial purpose and the vast majority (80\%) reported that disclosure of such information could influence their decisions about participating in research in the future. Said one participant: "They should know; the person should know the purpose of the study. You have Ambien 12 (hours) and Ambien CR (time release) and so they got the patent extended with the CR. They are looking at whether a formula makes a difference - and I think the study participant should be told exactly 
what is going on. It's coercion otherwise. We are looking at the formulary tweak. You have to be honest. If your study is not valid or fair or if you can't tell the truth, rethink the study. You have to know the real purpose." (P25). Said another: "Patents. Sure. Absolutely, for sure. I absolutely want to know. And I have a pretty high awareness of that issue and some of the drugs I have to take are still under patent and the costs are dramatically different from the generic drugs that I take." (P28) Among those who said it would not influence their decisions about participation, they still felt they should be informed about such issues.

Similarly, most participants $(84 \%)$ wanted to be fully informed about researcher compensation. The tacit assumption among participants seemed to indicate a belief that the conduct of and participation in research studies included some level of altruism or mutuality on the part of both the researcher and the participant. One participant deliberated in this way on that topic: "I would think twice about a study if there was an enrollment or other [financial] incentive for the researcher. It would depend on the amount and if they could use tissue, blood or psychological profile for long term benefit. I would feel deceived if someone was getting an enrollment or other incentive and not tell me. It would make a difference who was funding the study. More information should be provided - a statement like compensation for research - it is misleading because you think it [what the researcher is getting] is on par with what is happening to you."(p8) Another one said: "Yeah. That is pretty important information. Probably. Especially if there was a lot of compensation you might think twice about wanting to join. You would want to know if the doctor is doing it for the money. I would want to know if he is getting five grand for putting me through some study."(p2) The importance of transparency was expressed by this participant: "I may be less inclined if I thought there was a possible COI - that whoever was funding it stands to benefit from the findings. If Pfizer was doing a study to show that their drug is the hottest thing on the market. I don't have time for that kind of study. I would not participate; I would have no time for that. I can understand the benefit of running trials and trying to involve patients. I would want to know if my primary physician asked me, I would try to find out: OK could there be a benefit to my care provider by making me participate or framing data. And so if I started to feel pressure like someone really encouraging me, I would say no. I would immediately go into the terrible-two mode. If you are trying to push me...no thank you, I don't want it. I have no time to sort through the potential conflicts." (p5) "Wow. We didn't know that - they get paid for helping to run these studies? Really? Yes. I would want to know. I didn't realize they might get paid. Like my cancer nurse, is she paid by the cancer research foundation or how? Yes. I think you should know that. They should tell you. Who is getting paid and who is running the study and the whole reason why they are doing it. It is a matter of respect." (p47)

Willingness to join future research - When asked if they would be willing to participate in future studies $89 \%$ said they would join another study. Of those, $61 \%$ said they would join only under certain conditions. The "yes-but" group offered statements like: "I feel there are caveats." (P14); "I would if I could get all of the information beforehand. Then I could make an informed decision. I could be thinking it over. I never even realized that doctors do research in their clinics." (P7); "Well, it would depend on what the benefit would be and how much information I would be given. I am not adverse but I would now ask more questions." (P10); and "Yes but with a great deal more curiosity about the sponsors and the purpose of the study." (P19) There were also a few participants who expressed the view that if they had been put in the placebo or comparison group they would have withdrawn from the study. Said one mother whose child was participating in a behavioral study: "[We] will continue only if we are randomized to the telephonecontact therapy (with me, as opposed to face-to-face meetings with [my child]. The study is a comparison between these two modalities." (p15)

Although most participants seemed relatively satisfied with their experiences of participating in research there were some for whom the experience was less than expected. Indeed all of those who said they would not participate in another study also reported negative experiences with their research participation. It appears that if a person had a less than optimal experience when participating in the research, it led to an expression of what could have been done better and what additional information could have been provided. Indicative of this perspective, one participant said: "I would like to know the results of other trials; is it totally new or are there some results? And I would like to know the difference between this stent and others. And I would like to know the benefits - what are you [the participant] going to get out of this... [is it] attention and follow-up? I think that is all part of the disclosure." (p10) Another commented: "I mean thinking about this experience - I would have liked to have a better understanding of how I was going to feel. So even though it didn't have permanent effects that I know of - other than bad memory - I would have liked to know what it was going to be like. I don't recall having that described. Not to the degree of what I would experience." (p4)

Almost all of the participants expressed a desire to receive study results. Many expressed that some form of closure would have been appropriate. When asked if there were surprises, many did say that they were disappointed that they had not been re-contacted and informed about study results as was promised or anticipated when enrolling. The participants reported that the lack of such information dampened their willingness to participate in future studies.

\section{Discussion}

The findings from this study suggest that the guides that regulate the informed consent process - particularly the informed consent forms - may not be well aligned with the ways in which human subjects make decisions about participating in research. While claiming to have read the consent forms, most participants in this study had disregarded or discounted much of the information contained in them. The required information about sponsorship/funder, full purpose of the study, risks, benefits, confidentiality and privacy was marginally registered if the research seemed "legitimate." Key information in the consent forms was also disregarded or underestimated when the reason for participation was motivated by disease or some other personally compelling issue such as monetary reimbursement, an opportunity for the newest treatment or a "good deal" such as getting a flu shot for free. The participants seemed to make their decisions about participation based on intervening factors related to where the research is conducted (legitimacy of the setting), who conducts it (skilled clinician or colleague), and the relationship with or perceptions of the researcher (high levels of trust). When the research is offered in a clinic or other medical setting, participants appear to presume that the doctors or investigators know a great deal about the research and have considered all of the risks so that the participants will not be harmed. Thus even well-educated participants who stated that they read the informed consent documents for clinical studies seemed to make decisions based on their expectation of obtaining optimal treatment, the miracle drug, or the new gold standard. Participants did not fully understand that the study drugs may never make to it to the market, may be prohibitively expensive and so inaccessible to most people, or may have very serious 
side effects or consequences. When enrolling in non-clinical studies participants often reported an obligation to participate so as not to disappoint, or jeopardize the relationship with the researcher.

Indeed, there was such trust in the system and in the safety of participation that subjects often perceived that there was a greater personal risk associated with receiving either a placebo or the currently approved standard of care. If suspecting that they would be put in the placebo arm or existing treatment arm, some participants noted that they might even pull out of a study, a decision that could jeopardize randomization and so undermine the research integrity. Even those familiar with other types of research did not seem to transfer that knowledge when entering the medical realm via a clinical trial. What is surprising and deserving of attention when designing future policies for human subjects protection is that these findings consistently emerged even though this study was conducted with well educated, older people who would not have been deemed "vulnerable" or "at risk" in terms of their ability to read consent forms, understand the study, or make informed decisions.

These findings suggest that current approaches for protecting human subjects, some of which include requiring that consent forms contain more information and more detail, are written at ever lower reading levels, or use checklists or videos to ascertain informed consent may fail to overcome the beliefs and expectations that the participants bring to the research enterprise. In part, participants may be conditioned by western society to expect and ignore all sorts of cautionary language. There are warnings if you get on a rollercoaster, visit a ski hill, or walk along a nature path. Televised pharmaceutical advertisements extol the benefits of the newest drug and then follow the claim with a long list of potential side effects. When arriving for a medical appointment, patients are quickly given HIPAA and other privacy-related forms; in response, with a quick glance, patients often ask where they are supposed to sign. This disregard of warnings seems to be carried over to the consent process where the desire to participate seems to override serious appreciation of risk.

This kind of human behavior can also be understood by the theory of cognitive dissonance that suggests that individuals tend to seek consistency among their cognitions (i.e., beliefs, opinions) [36]. When there is an inconsistency between attitudes or behaviors (dissonance), something must change to eliminate the dissonance. In the case of a discrepancy between attitudes and behavior, it is most likely that the attitude will change to accommodate the behavior. There are three ways to eliminate dissonance: (1) reduce the importance of the dissonant beliefs, (2) add more consonant beliefs that outweigh the dissonant beliefs, or (3) change the dissonant beliefs so that they are no longer inconsistent [37]. Dissonance occurs most often in situations where an individual must choose between two incompatible beliefs: believing on one hand that research participation may have risks versus believing on the other hand that your clinician would not have suggested participation if the activity was risky. So in some respects, the extent to which the participants discounted information in the consent form, or even the process itself, is not surprising. Thus it is not more information in simplified language that matters when inviting participants to join a research study, but conveying the right information in ways that helps participants better understand how the goal of research and the goal of clinical care may be different from one another. Only when participants started thinking about issues like researcher/institutional compensation and commercial purposes of research such as obtaining patents or obtaining market shares did they begin to discern that there might be a difference between the goal of a particular research study and the goal of optimal individual care. Such recognition seemed to lead to a sort of "aaaha moment" whereby the participants then seemed to view issues such as receiving optimal personal care or not disappointing their doctor in a different light.

These findings suggest that if we truly want to enroll enlightened volunteers, we have to find ways to communicate in practical terms the actual purpose of the study, the results of past studies, what the investigator knows about the research intervention, and what the investigator is being paid for helping carry out the research. Any strategies that would help the participants clarify the differences between the goals of research and the goals of personal care deserve further exploration. Our study did not explore how such information should be provided, or how it could influence enrollment. Thus more research is certainly warranted. We need to better understand the range of factors and beliefs that participants bring to their decision making. We also need to better understand how human subjects will experience greater transparency about the overall research process.

\section{Acknowledgements}

This research was possible through a grant from the National Science Foundation, Award number SES-0821751. The grant was entitled: Exploring the consent process: Perspectives of IRB members and rural research participants.

\section{References}

1. Cook A, Hoas, $H$ (2011) Protecting research subjects: IRBs in a changing research landscape. IRB Ethics and Behavior 35: 14-19.

2. Cook A, Hoas $H$ (2011) Exploring the obligation to inform: Disclosing the purpose and benefits of research in an increasingly commercial research environment. AJOB-PR 2: 34-41.

3. Office of Human Subjects Research. The Belmont Report: Ethical principles and guidelines for the protection of human subjects of research. Access on $1 / 10 / 08$

4. Office for Human Research Protections. Federal policy for protection of human subjects. Accessed on 1/10/08

5. Department of Health and Human Services. The Health Insurance Portability and Accountability Act of 1996 (HIPAA) Privacy Rule.

6. Bosk CL (2001) Irony, ethnography and informed consent. In Barry Hoffmaster (ed) Bioethics in Social Context. Philadelphia, PA: Temple University; 212

7. Appelbaum PS, Grisso T (2001) MacArthur competence assessment tool fo clinical research (MacCAT-CR). Sarasota, FL: Professional Resource Press.

8. Iltis A. (2006) Lay concepts in informed consent to biomedical research: The capacity to understand and appreciate risk. Bioethics 20: 180-190.

9. Sugarman J, McCrory DC, Powell D, Krasny A, Adams B, et al. (1999) Empirical research on informed consent: An annotated bibliography. The Hastings Cent Rep 29: 1-42.

10. Stead M, Eadie D, Gordon D, Angus K (2004) Hello, Hello - it's English I speak: A qualitative exploration of patients' understanding of the science of clinical trials. J Med Ethics 31: 664-669.

11. Wray RJ, Stryker JE, Winer E, et al. (2007) Do cancer patients fully understand clinical trial participation? A pilot study to assess informed consent and patient expectations. Journal of Cancer Education. The Official Journal Of The American Association For Cancer Education Spring 22: 21-24.

12. Ormand KE (2009) Assessing the understanding of bio-bank participants American Journal of Medical Genetics 149:188-198.

13. Kass NE, Maman S, Atkinson J (2005) Motivations, understanding, and voluntariness in international randomized trials. IRB 27: 1-8.

14. Roberts LW (2002) Informed consent and the capacity for voluntarism. Am J Psychiatry. 159: 705-712.

15. Weinfurt KP, Dinan MA, Allsbrook JS, Friedman JY, Hall MA, et al. (2006) Policies of academic medical centers for disclosing financial conflicts of interest to potential research participants. Acad Med 81:113-118.

16. Weinfurt KP, Friedman JY, Dinan MA, Allsbrook JS, Hall MA, et al. (2006) Disclosing conflicts of interest in clinical research: views of institutional review 
Citation: Cook AF, Hoas H (2011) Trading Places: What the Research Participant Can Tell the Investigator about Informed Consent. J Clinic Res Bioeth 2:121. doi:10.4172/2155-9627.1000121

Page 7 of 7

boards, conflict of interest committees, and investigators. J Law Med Ethics 34: 581-591, 481.

17. Appelbaum PS, Lidz CW, Grisso T (2004) Therapeutic misconception in clinical research: Frequency and risk factors. IRB 26: 128.

18. King NMP, Henderson GE, Churchill LR, Davis AM, Hull SC, et al. (2005) Consent forms and the therapeutic misconception: The example of gene transfer research. IRB 27: 1-8.

19. Huntington I, Robinson W (2007) The many ways of saying yes and no: Reflections on the research coordinator's role in recruiting research participants and obtaining informed consent. IRB 29: 6-10

20. Ittis A (2006) Lay concepts in informed consent to biomedical research: The capacity to understand and appreciate risk. Bioethics 20: 180-190.

21. Appelbaum PS, Grisso T (2001) MacArthur competence assessment tool for clinical research (MacCAT-CR). Sarasota, FL: Professional Resource Press.

22. Appel JM (2001) Research guidelines: Changes urged. J Law Med Ethics 29: 103-04.

23. Shamoo AE, Schwartz J (2007) Universal and uniform protections of human subjects in research. Am J Bioeth 7: 7-9.

24. CenterWatch (2005) An industry in evolution. Boston: Center Watch.

25. Fisher JA (2006) Coordinating ethical clinical trials: The role of research coordinators in the contract research industry. Sociology of Health and IIIness 28: 678-694.

26. Fisher JA (2007) Ready to recruit or ready to consent populations? Qualitative Inquiry 13: 875-894.
27. Shuchman M (2007) Commercializing clinical trials-Risks and benefits of the CRO boom. N Eng J Med 357: 1356-1368.

28. Strauss AL, Corbin JM (1998) Basics of qualitative research: Techniques and procedures for developing grounded theory. 2nd ed. Thousand Oaks, CA Sage Publications, Inc; 1998.

29. Strauss AL, Corbin JM, (eds) (1997) Grounded theory in practice. 1st ed Thousand Oaks, CA: Sage Publications, Inc.

30. Creswell JW (1998) Qualitative inquiry and research design: Choosing among five traditions. Thousand Oaks, CA: Sage Publications, Inc.

31. Johnson, TP (2005) Snowball Sampling. Encyclopedia of Biostatistics. New Jersey: John Wiley \& Sons.

32. Morgan D L, Krueger R A (1997) Qualitative Data Analysis: An Expanded Sourcebook (2nd ed.). Thousand Oaks, CA: Sage.

33. Bogdan RC, Biklen SK. Qualitative Research for Education. An Introduction to Theory and Methods. Needham Heights, MA: Allyn and Bacon, 2006.

34. Cook. A \& Hoas, H. (2011) Protecting research subjects: IRBs in a changing research landscape. IRB 35: 14-19.

35. Cook A, Hoas H (2011) Exploring the obligation to inform: Disclosing the purpose and benefits of research in an increasingly commercial research environment. AJOB-PR 2: 34-41.

36. Festinger, L (1957) A Theory of Cognitive Dissonance. Stanford, CA: Stanford University Press.

37. Festinger, L Cognitive Dissonance, accessed on October 17, 2011. 